\title{
Réseaux thématiques de recherche avancée De nouveaux outils pour la recherche
}

\author{
Jean-Louis Pautrat
}

Directeur de la Fondation Nanosciences aux limites de la nanoélectronique (jusqu’au 31/12/2008).

Dans le sillage de la loi

"Pacte pour la recherche »,

votée en avril 2006,

le gouvernement a créé

deux types de structures

destinées à stimuler la

recherche française :

les Réseaux thématiques

de recherche avancée (RTRA)

et les Fondations

de coopération scientifique,

qui leur sont associées.

Parmi les treize RTRA créés,

deux relèvent très largement

de la physique :

« Nanosciences aux limites de

la nanoélectronique » localisé

à Grenoble, et « Triangle de

la Physique » localisé sur

Palaiseau, Orsay et Saclay.

\section{Qúest-ce qu'un RTRA ? Qúest-ce qủune Fondation de coopération scientifique?}

Un Réseau thématique de recherche avancée (RTRA) est un programme de coopération au sein d'un ensemble de laboratoires, généralement rassemblés sur un site donné. L'objectif de la création de ce réseau : viser à l'excellence, lutter contre l'éparpillement des moyens, accroître la visibilité des recherches menées.

Pour renforcer leur collaboration au sein d'un RTRA, les institutions responsables de ses différents laboratoires décident de s'unir au sein d'une Fondation de coopération scientifique. La Fondation est une structure de droit privé associée au réseau, et qui lui fournit les moyens de son action. La Fondation reçoit des financements sous forme d'un capital initial alloué par l'État et de contributions annuelles versées par les fondateurs.

Les Fondations de coopération scientifique sont autorisées à consommer leur capital initial dans la limite de 18\% par an, ce qui leur donne au départ au moins 5 ans de visibilité budgétaire. Au-delà, leur pérennisation peut être assurée par des dons publics (par les collectivités locales, notamment) ou privés (industriels ou particuliers), qu'elles sont habilitées à recevoir. La recherche de ces nouvelles sources de financement constitue un vrai défi.

Il est à noter que les différents RTRA se situent clairement dans la sphère de la recherche fondamentale, tout en restant étroitement connectés aux domaines applicatifs proches.

\section{Missions et modes d'action}

Un RTRA a pour mission d'apporter des moyens complémentaires aux unités de recherche partenaires dans le cadre d'une stratégie commune. L'objectif est de renforcer leur interactivité scientifique, leur rayonnement international et de leur permettre d'attirer les meilleurs scientifiques mondiaux. Un RTRA peut accorder des subventions et recruter des personnels qu'il met à la disposition des laboratoires.

Dès leur origine, les RTRA ont été conçus pour remédier à un certain nombre de lourdeurs des procédures actuelles de gestion de la recherche publique dans des domaines identifiés : réactivité trop faible des procédures de financement de projets, difficulté d'attirer de brillants chercheurs étrangers, montée en puissance trop lente des moyens dont disposent les jeunes chercheurs... Pour résoudre certaines de ces difficultés, les RTRA ont plusieurs modes d'action, dont voici les plus importants.

\section{Chaines d'excellence}

Elles permettent d'accueillir des chercheurs se distinguant au niveau international. Leur arrivée peut être accompagnée de contrats de doctorant et/ou post-doctorant, de crédits de fonctionnement et d'équipement. Les titulaires se trouvant dans l'impossibilité de séjourner continûment peuvent bénéficier de contrats leur permettant de venir plusieurs années de suite pour des durées limitées (1 à 3 mois).

\section{Contrats de doctorant}

Ils sont attribués sur projet et réservés à d'excellents candidats formés à l'étranger, qui ne peuvent pas bénéficier par ailleurs d'allocations ministérielles.

\section{Soutien aux nouveaux entrants}

Il a pour but d'aider au démarrage de nouvelles activités de recherche menées par des chercheurs nouvellement recrutés et permet le financement de dépenses de fonctionnement et d'équipement, ainsi qu'une bourse de thèse.

\section{Soutien aux plates-formes technologiques}

Les plates-formes d'un RTRA peuvent bénéficier de projets ciblés d'équipement et d'aide au fonctionnement. Ce soutien permet de développer la concertation entre tous les acteurs du site et de centrer les efforts sur des équipements structurants, en mettant en place les structures de gestion collective de ces équipements.

\section{Gouvernance}

Une Fondation de coopération scientifique est dirigée par un Conseil d'administration constitué de représentants des membres fondateurs et de personnalités qualifiées. Il est assisté 


\section{Organisation de la recherche}

d'un Conseil scientifique. Il nomme également un Comité de pilotage, constitué de scientifiques représentant l'ensemble des composantes du RTRA. Ce comité veille à la cohérence et à la qualité des recherches menées dans le réseau. Parmi les projets déposés, il propose à la Fondation ceux dont l'excellence est attestée par les expertises internationales qu'il a commandées et qui sont en mesure de renforcer la cohérence du réseau de laboratoires.

Le Conseil d'administration nomme un directeur, qui a en charge la gestion administrative de la Fondation ainsi que la direction du Comité de pilotage.

La vie des Fondations de coopération scientifique est étroitement contrôlée par l'État.

\section{Le RTRA sera-t-il un bon outil au service de l'efficacité de la recherche? \\ ou hien une usine à gaz dont le fonctionnement complexe ne donnerd satisfaction à personne?}

Il est bien sûr trop tôt pour juger de l'efficacité des RTRA et de leur impact sur les recherches menées. Cependant, tous les acteurs au sein de ces structures ont la ferme volonté d'en faire un instrument souple et réactif au service de la recherche. Déjà, après un peu plus d'une année d'existence, cette structure a démontré son utilité. Les projets soumis aux RTRA ont, dans leur majorité, cherché à répondre aux ambitions affichées : attirer des chercheurs de qualité, développer les coopérations entre les laboratoires et entre les disciplines, développer des outils technologiques et des instruments de caractérisation, et, enfin, associer recherche fondamentale et technologie. La rationalisation des moyens technologiques, la simplification des conditions d'accès à ces moyens, le regroupement des moyens de caractérisation ont aussi été fortement encouragés. Bien sûr, ces évolutions étaient déjà souhaitées par de nombreux chercheurs. Le RTRA n'a peut-être été que le catalyseur de cette évolution. Tous ces signes semblent en tout cas promettre la réussite.

\section{À Grenoble : Nanosciences aux limites de la nanoélectronique}

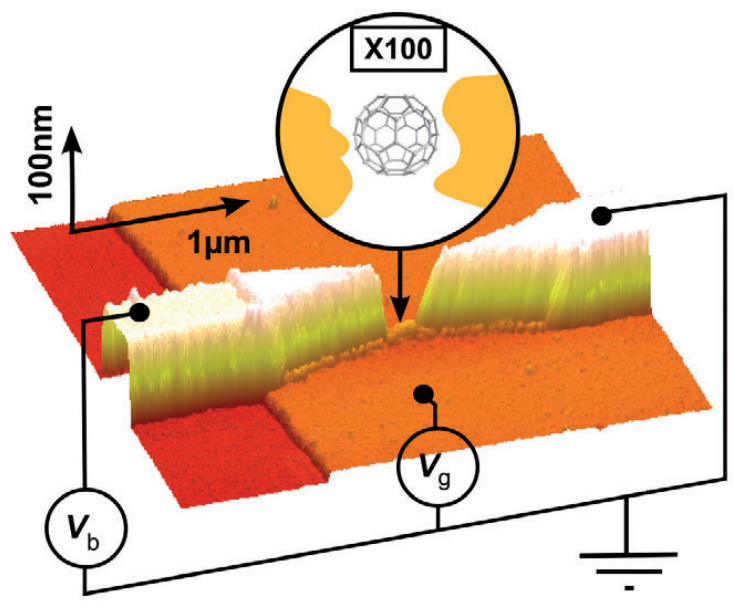

Nanojonction comportant une molécule de fullérène entre deux électrodes.
La Fondation et le RTRA « Nanosciences aux limites de la nanoélectronique » sont les seuls en France à se consacrer exclusivement à cette thématique. Ses quatre membres fondateurs, le CEA, le CNRS, l'Université Joseph Fourier et le groupe Grenoble INP, sont des acteurs reconnus des nanosciences. Ils souhaitent, par ce biais, dynamiser la recherche en nanosciences en mettant en commun leurs connaissances, en mutualisant leurs outils, en fédérant leurs initiatives autour d'équipes communes. En effet, la collaboration entre les quatre membres fondateurs de la Fondation Nanosciences est depuis longtemps inscrite dans les faits et se traduit notamment par l'existence de nombreux laboratoires mixtes. La structuration de ces collaborations au sein d'un Réseau thématique de recherche avancée va accélérer et renforcer cette intégration.

\section{Pourquoi une telle fondation à Grenoble ?}

La tradition universitaire de la ville de Grenoble, la présence d'organismes publics de recherche (CEA, CNRS...) et les grands noms de l'industrie de pointe (STMicroelectronics, Soitec, Schneider...) qui y ont élu domicile ou y sont nés ont fait de la région grenobloise un périmètre de référence en recherche technologique, en France et en Europe. Dans ce contexte, on ne peut oublier l'apport si fructueux de Louis Néel, prix Nobel de physique en 1971. Le fort potentiel de recherche fondamentale et technologique de Grenoble s'est naturellement tourné vers les nanosciences et les nanotechnologies.

Grenoble a très tôt pris le virage des nanotechnologies et bénéficie d'une longue expérience dans le domaine. La création en 2006 du pôle d'excellence
Roland Hérino,

Directeur de la Fondation

23 rue des martyrs, 38000 Grenoble roland.herino@fondation-nanosciences.fr www.fondation-nanosciences.fr 
$>>$

européen en micro et nanotechnologie MINATEC ${ }^{\circledR}$ dans la capitale des Alpes, en fait le lieu de référence des nanosciences au sein de l'Union européenne. L'émergence du pôle de compétitivité MINALOGIC a confirmé l'engagement des acteurs de l'innovation industrielle. La création de la Fondation Nanosciences à Grenoble a donc été un mouvement naturel, un choix logique qui permet de compléter le dispositif de recherche et d'innovation en Rhône-Alpes.

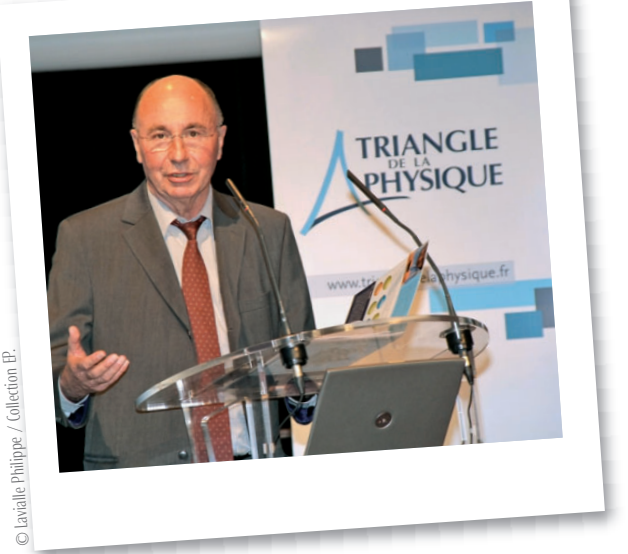

Christian Colliex, directeur du Triangle de la Physique, lors de l'inauguration du 19 mai 2008 à l'École polytechnique, Palaiseau.

\section{-Vie scientifique du Triangle de la Physique}

La recherche au Triangle de la Physique est structurée autour de sept thèmes fédérateurs

- Cohérence et intrication quantiques,

- Matière hors d'équilibre : de la molécule aux nanoparticules,

- Matière complexe,

- Matière à fortes corrélations quantiques,

- Électronique de spin,

- Pôle lumière extrême,

- Nanophotonique,

et de trois axes transversaux :

- Instrumentation à ses limites,

- Théorie, calculs numériques,

- Synthèse de nouveaux objets.

Elle fait l'objet d'une lettre électronique en anglais, téléchargeable sur le site.

Christian Colliex, Directeur, chercheur CNRS, Laboratoire de physique des solides-Orsay, christian.colliex@triangledelaphysique.fr

\section{Élisabeth Bouchaud,}

Directrice adjointe, chercheuse, CEA-Saclay, elisabeth.bouchaud@triangledelaphysique.fr

www.triangledelaphysique.fr

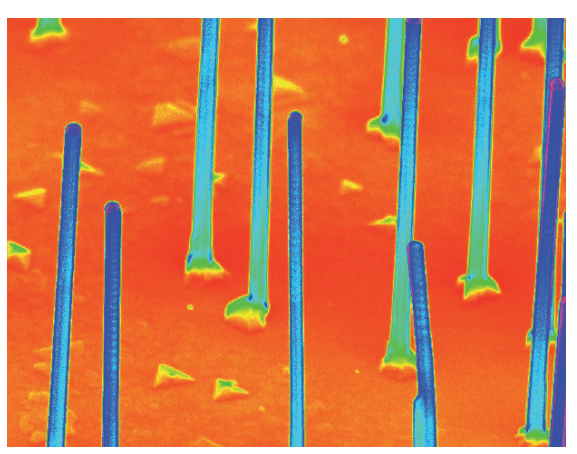

Les équipes soutenues par la Fondation se consacrent aux thèmes suivants :

- Nanoélectronique quantique,

- Nanomagnétisme et électronique de spin,

- Nanophotonique,

- Électronique moléculaire,

- Nanomatériaux, nanobonding,

nanostructuration,

- Nanocaractérisation et métrologie,

- Vivant aux limites de la nanoélectronique,

- Nanomodélisation.

\section{Le Triangle de la Physique,}

\section{un réseau mis en place par et pour les physiciens}

du Plateau de Saclay

Plus de mille physiciens, rattachés à trente-six laboratoires sur les trois campus d'Orsay, de Palaiseau et de Saclay, regroupés autour de sept thèmes fédérateurs de la physique quantique à la lumière extrême en passant par la nanophysique et la matière fortement corrélée, ont entrepris de construire et de mettre en place ce Réseau thématique de recherche avancée (RTRA), créé par décret en date du 23 mars 2007.

Huit organismes fondateurs se sont associés pour accompagner cette création : le CNRS, le CEA, l'Université Paris-Sud, l'École polytechnique, Supélec, l'Institut d'Optique-graduate school, l'ENSTA et l'ONERA.

Les objectifs du Triangle de la Physique sont de mieux utiliser les compétences déjà rassemblées, d'amplifier la visibilité des résultats de la recherche qui y est menée et d'accroître l'attractivité internationale. À toutes les étapes, de l'élaboration de projets à la sélection, la diversité et la complémentarité des compétences et des chercheurs impliqués permettent de favoriser l'émergence et la cohésion du réseau. Ce dernier est un outil d'action privilégié pour faire évoluer le paysage scientifique et lui procurer une réactivité indispensable. Dans un paysage scientifique propice à des évolutions de plus en plus rapides, le Triangle de la Physique peut également apporter son soutien critique pour monter des structurations ou des collaborations nouvelles.

\section{Qúen est-il un an après sa naissance?}

Le Triangle de la Physique évolue graduellement vers un type de «superlaboratoire virtuel ", transcendant les frontières interorganismes pour élaborer une politique scientifique concertée à l'échelle du territoire du plateau de Saclay.

Le réseau permet de construire de nouveaux instruments, de développer les outils théoriques d'interprétation et de simulation et de créer de nouveaux objets d'études.

L'attribution du Prix Nobel de Physique à Albert Fert pour ses travaux antérieurs sur la magnétorésistance géante, a démontré au tout premier rang mondial la qualité d'un des chercheurs ayant participé au montage du Triangle de la Physique et l'importance stratégique d'une thématique fédératrice du réseau, la spintronique. Que ce premier succès soit suivi par de nombreux autres prix ou reconnaissances individuels ou collectifs, mais aussi, par exemple, par l'attraction vers le plateau de Saclay de nouveaux centres de recherches privés ou publics, nationaux ou internationaux, c'est la motivation la plus profonde du Triangle de la Physique. 